\title{
Audio spectrum and sound pressure levels vary between pulse oximeters
}

\author{
[Le spectre audible et les niveaux de pression sonore varient entre \\ sphygmo-oxymètres]
}

Deven Chandra MD, * Michael J. Tessler MD, $†$ John Usher BEngf

Purpose: The variable-pitch pulse oximeter is an important intraoperative patient monitor. Our ability to hear its auditory signal depends on its acoustical properties and our hearing. This study quantitatively describes the audio spectrum and sound pressure levels of the monitoring tones produced by five variable-pitch pulse oximeters.

Methods: We compared the Datex-Ohmeda Capnomac Ultima, Hewlett-Packard MII66A, Datex-Engstrom AS/3, Ohmeda Biox 3700, and Datex-Ohmeda 3800 oximeters. Three machines of each of the five models were assessed for sound pressure levels (using a precision sound level meter) and audio spectrum (using a hanning windowed fast Fourier transform of three beats at saturations of $99 \%, 90 \%$, and $85 \%$ ).

Results: The widest range of sound pressure levels was produced by the Hewlett-Packard MII66A (46.5 \pm I.74 dB to $76.9 \pm 2.77 \mathrm{~dB})$. The loudest model was the Datex-Engstrom AS/3 $(89.2 \pm 5.36 \mathrm{~dB})$. Three oximeters, when set to the lower ranges of their volume settings, were indistinguishable from background operating room noise. Each model produced sounds with different audio spectra. Although each model produced a fundamental tone with multiple harmonic overtones, the number of harmonics varied with each model; from three harmonic tones on the Hewlett-Packard MII66A, to 12 on the Ohmeda Biox 3700. There were variations between models, and individual machines of the same model with respect to the fundamental tone associated with a given saturation.

Conclusion: There is considerable variance in the sound pressure and audio spectrum of commercially-available pulse oximeters. Further studies are warranted in order to establish standards.
Objectif : Le sphygmo-oxymètre à tonalité variable est un moniteur peropératoire important. Notre capacité à entendre son signal sonore dépend de ses propriétés acoustiques et de notre audition. Notre étude quantitative décrit le spectre audible et les niveaux de pression sonore des tonalités de monitorage produites par cinq sphygmo-oxymètres à tonalité variable.

Méthode : Nous avons comparé les oxymètres suivants : DatexOhmeda Capnomac Ultima, Hewlett-Packard MII66A, DatexEngstrom AS/3, Ohmeda Biox 3700 et Datex-Ohmeda 3800. Trois appareils de chaque modèle ont été évalués pour les niveaux de pression sonore avec un sonomètre de précision, et pour le spectre audible en utilisant une transformée de Fourier à fenêtre Hanning de trois battements à des saturations de $99 \%, 90 \%$ et $85 \%$.

Résultats: La plus grande étendue de niveaux sonores a été produite par le Hewlett-Packard MII66A (46,5 \pm I,74 dB à 76,9 $\pm 2,77 \mathrm{~dB}$ ). Le Datex-Engstrom AS/3 a produit la plus haute intensité sonore $(89,2 \pm 5,36 \mathrm{~dB})$. Trois oxymètres, dont les paramètres de volume étaient fixés à de bas niveaux, ne pouvaient être entendus dans le bruit de la salle d'opération. Chaque modèle a produit des sons au spectre audible différent ayant un son fondamental et de multiples partiels harmoniques. Mais le nombre d'harmoniques variait selon le modèle, allant de trois pour le Hewlett-Packard MII66A à I 2 pour le Ohmeda Biox 3700. II y avait des variations entre les modèles et entre les appareils d'un même modèle quant au son fondamental associé à une saturation donnée.

Conclusion : Il y a une grande variation de pression sonore et de spectre audible pour les sphygmo-oxymètres offerts sur le marché. II reste maintenant à fixer des normes.

From the Department of Anesthesia, McGill University; ${ }^{*}$ the Department of Anesthesia, $†$ Sir Mortimer B. Davis-Jewish General Hospital, and McGill University; and the Faculty of Music, Multichannel Audio Research Laboratory, McGill University, Montréal, Québec, Canada. Address correspondence to: Dr. Michael J. Tessler, Room A-335, Department of Anesthesia, Sir Mortimer B. Davis-Jewish General Hospital, 3755 Cote-Ste-Catherine Road, Montréal, Québec H3T 1E2, Canada. Phone: 514-340-8222, ext. 5701;

E-mail: mtessler@ana.jgh.mcgill.ca

There were no funding sources for this paper.

Assessed January 4, 2005.

Accepted for publication June 2, 2005.

Revision accepted July 27, 2005. 
S INCE its introduction in 1983 by the Nellcor Corporation (Hayward, CA, USA), the variable-pitch pulse oximeter has rapidly become a common patient monitoring device in many clinical settings. A variable pitch oximeter is especially valuable in the intraoperative environment because it provides continuous auditory monitoring of heart rate and oxygen saturation without the clinician even having to see the monitor itself. Most people are able to perceive the pitch change on a variable-pitch pulse oximeter, ${ }^{1}$ and variable-pitch oximeters significantly reduce the response time of anesthesiologists to an adverse event. $^{2}$

There are many acoustical properties of operating rooms, and variable-pitch pulse oximeters, which may influence continuous auditory monitoring by anesthesiologists. Several of these properties have been researched previously. ${ }^{3-7}$ For example, noise levels in the operating room have been systematically measured $^{4-7}$ as has the hearing acuity of anesthesiologists. ${ }^{8}$ However, an audio spectrum and sound pressure level analysis of the monitoring beeps produced by common variable-pitch pulse oximeters has not been reported. The objective of this study was to quantitatively describe the audio properties of five variable-pitch pulse oximeters in the operating room environment.

\section{Acoustics for anesthesiologists}

In order to follow a discussion on sound analysis, a rudimentary knowledge of acoustical theory is required. We therefore include the following definitions.

\section{Frequency of a sound 9}

The rate at which a mechanical disturbance is propagated through a medium (i.e., sound passing through air), measured in cycles per second, or Hertz $(\mathrm{Hz})$. This frequency determines the pitch of a sound (i.e., 261.63 $\mathrm{Hz}=$ the musical note known as "Middle C").

\section{Pitch $^{9}$}

That attribute of auditory sensation in terms of which sounds may be ordered on a musical scale.

\section{Audio spectrum ${ }^{10}$}

The frequency bandwidth which describes the human auditory spectrum. This frequency range extends over three orders of magnitude, from about 20 to 20,000 $\mathrm{Hz}$.

\section{Decibel $(d B)^{9}$}

The logarithmic scale used to describe sound pressure. Its logarithmic nature simplifies notation in that the ratio of sound pressures comparing the human threshold of hearing to the threshold of pain is approximately $2 \times 10^{-8}$ (i.e., 0.00000002 ) micropascals $(\mu \mathrm{Pa}$ ) to 2 $\times 10^{4}$ (i.e., 20,000$) \mu \mathrm{Pa}$ or simply, 0 to $120 \mathrm{~dB}$.

The word "decibel" is made up of the metric prefix "deci", and the unit "bel". This unit was named after Sir Alexander Graham Bell who originated the concept in order to simplify calculations of telegraph performance. In order to gain an appreciation for these values, consider the following examples of average noise levels: quiet living room $=40 \mathrm{~dB}$, operating room $=$ $77.32 \mathrm{~dB}(\mathrm{~A}),{ }^{6}$ rock concert $=108$ to $120 \mathrm{~dB}$.

Averaging of decibel measurements cannot be done by way of the arithmetic mean, due to their logarithmic nature. For instance a noise of $40 \mathrm{~dB}$ is not twice as intense as a noise of $20 \mathrm{~dB}$, but it is closer to nine times as intense. In other words, intensity increases about three times for every $10 \mathrm{~dB}$. Correct averaging of decibel measurements requires conversion of the decibel values into linear pressure ratios, taking the arithmetic mean of these pressure ratios, and then converting the average pressure ratio into decibels again.

" $A$ " Weighted $d B$, or $d B(A)^{9}$

A correction factor which adjusts $\mathrm{dB}$ level with respect to frequency, more accurately representing human perception of loudness. For instance, because human hearing is most acute between approximately 0.5 to 4 $\mathrm{kHz}$, humans will perceive a noise within this range as being louder than a noise outside of this range, even if in reality, these noises are both of equal sound pressure level.

\section{Resonances $^{10}$}

Overtones or harmonics are also called resonances. In the phenomenon of resonance, a system that vibrates at some natural frequency is subjected to external vibrations of the same frequency; as a result, the system resonates, or vibrates at large amplitude. Examples of acoustical resonance include the vibration induced in a violin or piano string of a given pitch when a musical note of the same pitch is sung or played nearby, or the folklore of opera singers being able to shatter wine glasses with forced vibration from their singing voices.

\section{Timbre ${ }^{11}$}

Timbre has been defined as "that attribute of auditory sensation in terms of which a listener can judge that two sounds, similarly presented and having the same loudness and pitch, are different". 


\section{Materials and methods Apparatus}

In this descriptive study, we compared the audio spectrum and sound pressure levels of five models of variable-pitch pulse oximeters. The study took place at two McGill University Teaching Hospitals, The Sir Mortimer B. Davis-Jewish General Hospital (SMBD-JGH), and the Royal Victoria Hospital $(\mathrm{RVH})$. The five models of pulse oximeters studied were: The Hewlett-Packard Ml166A (Palo Alto CA, USA), the Datex-Ohmeda Capnomac Ultima, the Datex-Engstrom AS/3, the Ohmeda Biox 3700, and the Datex-Ohmeda 3800 (all from divisions of the Instrumentarium Corporation, Helsinki Finland). Three machines representing each of the five models described above were selected, so that a total of 15 machines were studied. At the time of the study each machine had been in use for more than two years, and was in regular use by the anesthesia departments at each hospital. The same finger probe was used while recording both audio spectra, and sound level measurements on all three Hewlett-Packard oximeters. Likewise, the same finger probe was used on the remaining oximeters.

Sound pressure levels were measured with a Brüel \& Kjær (Nærum, Denmark) type 2235 precision sound level meter with type 4176 microphone. Digital recordings for audio spectrum analysis were made using a Labtec (Vancouver, WA, USA) unidirectional, universal serial bus, digital microphone, and notebook personal computer. Microsoft Windows XP "sound recorder" software was used to record mono, 16 bit, $48 \mathrm{kHz}$.wav format files.

\section{Procedure for sound level measurements}

Sound pressure levels were measured in quiet, unused operating rooms (usually during the evenings) when background noise was minimal. All other monitors, and anesthesia equipment were turned off - leaving the operating room ventilation fans as the only background noise. A total of six operating rooms were used, of approximately similar dimensions, and background noise levels.

Measurements were recorded from a standardized position similar to where an anesthesiologist would sit while monitoring a case intraoperatively, approximately $0.5 \mathrm{~m}$ in front of the anesthesia machine, and at a height of approximately 0.9 to $1 \mathrm{~m}$. The sound level meter was gently waved through the air during measurements to avoid measuring resonant nodes within the rooms. The following sound level meter settings were used: sound incidence $=$ random, frequency weighting $=\mathrm{A}$, time weighting $=$ fast, display $=$ instan- taneous. The "random incidence" setting corresponds to an approximately omni-directional sensitivity of the sound level meter. As the orientation of the anesthesiologists' head is generally changing throughout an operation, this setting was more appropriate than a directional sensitivity setting. The integrating window of the sound level meter is $\sim 10 \mathrm{msec}$, which allows sufficient temporal resolution for loudness measurements of the pulse beeps, which are typically of 300 to 400 msec duration.

For each volume setting available (i.e., 1-10 on a Datex-Engstrom AS/3), 20 measurements were recorded, which consisted of 20 consecutive oximeter beeps (at $\mathrm{SpO}_{2}=97 \%$, with the standardized finger probe on the experimenter's finger). For instance, because a Datex-Engstrom AS $/ 3$ pulse oximeter has ten volume settings, and 20 measurements were recorded at each volume setting, there were 200 measurements for each machine of this model.

\section{Statistics and analysis of sound pressure level measure- ments}

The mean sound level for each volume setting of each model of pulse oximeter was calculated (Table I). These means were obtained using the procedure described above to obtain measurements. The following formula was used to calculate mean sound pressure levels: ${ }^{12}$

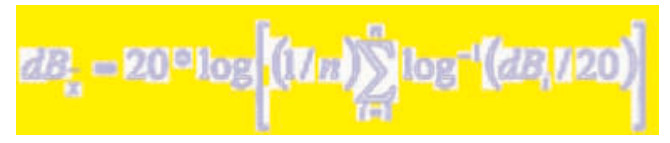

This formula is required because the decibel scale was designed as a logarithmic scale describing the ratio of a given sound pressure to the sound pressure (measured as watts $/ \mathrm{cm}^{2}$ ) at the threshold of human hearing $(0 \mathrm{~dB})$. Basically, the $\mathrm{dB}$ measurements were transformed into linear sound pressure ratios, averaged, and then converted back into logarithmic dBs. Standard deviations, and confidence intervals were calculated where appropriate.

\section{Procedure for audio spectrum analysis}

Digital recordings were taken from each of the 15 machines. All other monitors, and anesthesia equipment were turned off - leaving the operating room ventilation fans as the only background noise. A total of six operating rooms were used, all of approximately similar dimensions and background noise levels. The same volume setting was used for each model of oximeter. The unidirectional, digital microphone 
TABLE I Mean sound pressure levels $(\mathrm{dB}(\mathrm{A}))$ and SD

\begin{tabular}{|c|c|c|c|}
\hline Brand and model number & Volume setting & $\operatorname{Mean} d B(A)$ & $S D$ \\
\hline \multirow[t]{4}{*}{ Datex-Ohmeda Capnomac Ultima } & 1 & * & * \\
\hline & 2 & 51.1 & 1.26 \\
\hline & 3 & 55.0 & 1.89 \\
\hline & 4 & 59.4 & 1.44 \\
\hline \multirow{17}{*}{ Hewlett-Packard M1166A } & 15 & * & * \\
\hline & 30 & * & * \\
\hline & 45 & 46.5 & 1.74 \\
\hline & 60 & 49.4 & 2.32 \\
\hline & 75 & 53.6 & 3.91 \\
\hline & 90 & 56.8 & 5.08 \\
\hline & 105 & 59.5 & 5.15 \\
\hline & 120 & 61.6 & 5.30 \\
\hline & 135 & 63.9 & 4.63 \\
\hline & 150 & 65.7 & 4.45 \\
\hline & 165 & 66.5 & 4.73 \\
\hline & 180 & 67.9 & 4.27 \\
\hline & 195 & 69.9 & 3.65 \\
\hline & 210 & 72.3 & 3.50 \\
\hline & 225 & 73.7 & 3.30 \\
\hline & 240 & 75.1 & 3.45 \\
\hline & 255 & 76.9 & 2.77 \\
\hline \multirow[t]{10}{*}{ Datex-Engstrom AS /3 } & 1 & 59.7 & 1.18 \\
\hline & 2 & 63.1 & 1.66 \\
\hline & 3 & 65.6 & 1.71 \\
\hline & 4 & 70.8 & 1.44 \\
\hline & 5 & 74.1 & 1.44 \\
\hline & 6 & 76.2 & 1.31 \\
\hline & 7 & 78.3 & 1.43 \\
\hline & 8 & 81.0 & 2.17 \\
\hline & 9 & 83.9 & 2.97 \\
\hline & 10 & 89.2 & 5.36 \\
\hline \multirow[t]{10}{*}{ Ohmeda Biox 3700} & 1 & * & * \\
\hline & 2 & * & * \\
\hline & 3 & 43.5 & 0.723 \\
\hline & 4 & 45.2 & 0.885 \\
\hline & 5 & 48.0 & 1.04 \\
\hline & 6 & 50.9 & 1.55 \\
\hline & 7 & 51.5 & 0.721 \\
\hline & 8 & 52.4 & 0.993 \\
\hline & 9 & 54.2 & 1.21 \\
\hline & 10 & 58.2 & 1.26 \\
\hline \multirow[t]{5}{*}{ Datex-Ohmeda 3800} & 1 & 55.1 & 1.72 \\
\hline & 2 & 61.6 & 1.72 \\
\hline & 3 & 65.5 & 1.07 \\
\hline & 4 & 71.1 & 0.894 \\
\hline & 5 & 76.4 & 1.54 \\
\hline
\end{tabular}

* Indistinguishable from background noise. Mean $\mathrm{dB}(\mathrm{A})$ calculated as follows: $\mathrm{dB}_{\text {mean }}=20 \mathrm{x} \log \left[(\mathrm{l} / \mathrm{n}) \sum \log ^{-1}\left(\mathrm{~dB}_{\mathrm{i}} / 20\right)\right]$. Data are presented as mean decibel level $(\mathrm{dB}(\mathrm{A}))$ and standard deviation $(\mathrm{SD})$ for each model at every volume setting.

(described above) was placed directly adjacent to the speaker of each machine for all recordings.

Each recording consisted of three beats at saturations of $99 \%, 90 \%$, and $85 \%$ respectively. The recordings were made using the standardized finger probes (described above), and the experimenter's finger. The desired oxygen saturation levels were achieved with supplemental oxygen, or brief occlusions of the digi- tal pulse. One author (D.C.), over the course of the study, was able to reliably limit digital blood flow by pressing on the digital arteries to create a reproducible and sustainable result at the lower oxygen saturations, without loss of the audible signal. Data were recorded only after a steady-state ( ten consecutive beats), was achieved at a given saturation. 
Analysis of audio spectrum recordings

Spectral analysis of these recordings allows for a quantitative description of the timbre of noises produced by pulse oximeters. Analysis was conducted using a hanning windowed 1024-point, no overlap, fast Fourier transform (FFT) of each recording. The frequency resolution may be above that of the human auditory system ${ }^{13}$ (i.e., we will not be able to hear all the spectral detail implied by the FFT). However, this high resolution enables accurate determination of the harmonic frequencies present, allowing a thorough description of the timbre of each tone.

\section{Results}

The sound pressure level analysis results are presented in Table I. The quietest model was the Ohmeda Biox 3700 which was only $58.2 \pm 1.26 \mathrm{~dB}$ on its loudest setting $(10 / 10)$. The loudest model was the Datex-Engstrom AS $/ 3$, which was $89.2 \pm$ $5.36 \mathrm{~dB}$ on its loudest setting. The Datex-Ohmeda Capnomac Ultima, the Hewlett-Packard Ml166A, and the Ohmeda Biox 3700 had ranges of volume settings, some of which were indistinguishable from background noise.

The widest range of sound pressure levels was produced by the Hewlett-Packard Ml166A, which from its lowest to highest settings produced sound pressure levels from $46.5 \pm 1.74 \mathrm{~dB}$ to $76.9 \pm 2.77$ $\mathrm{dB}$ respectively.

The audio spectrum analysis results are presented in Table II. Some models generated the same fundamental frequencies (e.g., Hewlett-Packard M1166A and Datex-Ohmeda 3800) at some oxygen saturations, but no two models had the same frequency at all oxygen saturations. Further, the timbre was different among the models, and the number of harmonic tones varied from three with the Hewlett-Packard Ml166A, to 12 with the Ohmeda-Biox 3700.

\section{Discussion}

We have shown that pulse oximeters generate polytonal noises, and that there is variation in fundamental tones between models, even within models of pulse oximeters. In describing the sound pressure levels emitted from pulse oximeters, we also determined that many of these devices have volume settings which are indistinguishable from background operating room noise, and that some have settings loud enough to cause annoyance, and harm with extended exposure.

Audio spectrum analysis reveals that all pulse oximeter tones consist of polytonal noises. The DatexOhmeda Capnomac Ultima for example, produces polytonal beeps consisting of several simultaneous
TABLE II Fundamental frequencies of pulse oximeter tones $(\mathrm{Hz})$ at three oxygen saturations

\begin{tabular}{llll}
\hline Brand and model number & $\mathrm{SpO}_{2}=85 \%$ & $\mathrm{SpO}_{2}=90 \%$ & $\mathrm{SpO}_{2}=99 \%$ \\
\hline Datex-Ohmeda Capnomac & 844 & 891 & 1031 \\
Ultima & 844 & 891 & 1031 \\
Hewlett-Packard Ml166A & 891 & 938 & 1078 \\
& 469 & 516 & 703 \\
Datex-Engstrom AS/3 & 469 & 516 & 703 \\
& 849 & 516 & 703 \\
Ohmeda Biox 3700 & 844 & 891 & 984 \\
& 844 & 891 & 984 \\
& 422 & 469 & 584 \\
Datex-Ohmeda 3800 & 375 & 469 & 563 \\
& 469 & 422 & 469 \\
& 469 & 516 & 609 \\
\hline
\end{tabular}

Data are presented as the fundamental frequency for each machine of each model at $85 \%, 90 \%$, and $99 \%$ oxygen saturation.

tones per beep. Similar to the tones generated by a bell, this wide bandwidth noise allows pulse oximeter tones to be heard by individuals affected with auditory disorders, such as presbycusis.

Variable-pitch pulse oximeters generate tones which correspond to the patient's heart rate, and oxygen saturation. These tones rise or fall in pitch as the patient's oxygen saturation rises and falls. Audio spectrum analysis confirms this assertion, but also demonstrates that there is no standardization with respect to pitch and oxygen saturation between different pulse oximeter models. For instance, an oxygen saturation of $99 \%$ will produce a fundamental tone of $\sim 700 \mathrm{~Hz}$ on a Hewlett-Packard Ml166A oximeter. However, the same saturation produces a tone of $\sim 980 \mathrm{~Hz}$ on a Datex-Engstrom AS/3. Interestingly, careful examination of the data reveals that some oximeters do not demonstrate standardized pitches within one type of model. For instance, one Ohmeda Biox 3700 generated a tone of $\sim 422 \mathrm{~Hz}$ at a saturation of $85 \%$, whereas another machine of the same model generated a tone of $\sim 375 \mathrm{~Hz}$ at the same saturation. Our results on the tones generated are consistent with those of Santamore and Cleaver. ${ }^{3}$ They found, using simulator technology to generate oxygen saturation levels, that each of the different models of variable pitch pulse oximeters tested had different frequencies at the oxygen saturation levels measured. We used brief occlusions of the digital arteries to achieve the relevant oxygen saturations, which we think is more physiological. Interestingly, the tonal frequencies of 
the Datex-Ohmeda AS $/ 3$, the one model tested in both studies, were different at all saturations. ${ }^{3}$

Tonal differences between oximeters have clinical implications for anesthesiologists. A visual check of oxygen saturation when taking over a case, or transferring a patient to a different pulse oximeter, is part of a vigilant anesthesiologist's practice. However, a conscious visual and auditory check would allow the physician to accommodate to the particular tones of the new oximeter. Standardizing the tones of variable-pitch pulse oximeters would eliminate the need for a conscious auditory check, and might allow an anesthesiologist to approximate a patient's oxygen saturation without checking the digital display. This may be of particular benefit in critical situations such as airway emergencies, when a physician's attention is focused on the patient's airway, rather than the monitors.

Each model of pulse oximeter has a markedly different audio spectrum. These differences occur mainly in harmonic composition (Table II), which affects the perceived timbre ${ }^{12}$ and pitch of the tone. Because the human ear perceives five to eight harmonics of a complex sound, one might expect the pitch of a tone (and therefore the oxygen saturation) to be determined more easily from sounds with more harmonics.

Sound pressure level analysis revealed that three of the pulse oximeters have volume settings which do not provide sufficient loudness to be distinguishable from background noise in the operating room. Conversely, the loudest settings can provide a significant level of noise pollution.

In our analysis the oximeters were studied under relatively quiet conditions (an unused operating room), with ventilation systems as the only background noise. Despite these conditions, a volume setting of " $\mathrm{l}$ " on a Datex-Ohmeda Capnomac Ultima pulse oximeter was indistinguishable from background noise. This lack of loudness will be exacerbated in fully operational operating rooms which, as mentioned above, have been found to have noise levels of $\sim 77.32 \mathrm{~dB}(\mathrm{~A}){ }^{6}$ This requires the vigilant anesthesiologist to ensure that the loudness of the pulse oximeter is adjusted to overcome the ambient noise in a busy operating room, but not so loud as to contribute to noise pollution.

The loudest oximeter studied, the Datex-Engstrom AS $/ 3$, produced noises of $89.2 \mathrm{~dB}(\mathrm{~A})$ on average. This corresponds to the approximate loudness of a pneumatic drill, or household lawnmower. Prolonged exposures to noise levels of this magnitude are not only annoying to operating room personnel, but can be hazardous to their health. ${ }^{5}$

Though experimental design was controlled to the greatest extent possible, the analysis has several limita- tions. Perhaps the most significant is the fact that operating rooms were used, rather than a specialized room in an acoustic research facility which can standardize ambient noise. The analysis was carried out in two different hospitals, in six different operating rooms. However, all of operating rooms had similar dimensions and acoustical properties. Each monitor studied had been in daily use (weekdays) for more than two years, to reflect the response of the "real-world setting". We also chose not to perform a statistical comparison of the data between oximeter types because we tested three machines only of each model. The results are limited to those pulse oximeters and models available to the authors at the study institutions, and may not necessarily be generalized to other pulse oximeters available in modern anesthetic practice.

In summary, this study provides a quantitative analysis of the sound pressure levels, and audio spectrum of five common variable-pitch pulse oximeters. There is considerable variability in the sound pressure and audio spectrum of these devices which may have important clinical implications. Standardization of tones generated by various brands of oximeters might allow anesthesiologists to use these different brands with greater ease. Regulation of available volume settings should ensure that this critical auditory monitor can be heard above ambient noise in the operating room. This study invites further audio analysis of operating room equipment, and suggests the creation of standards for the auditory signals of pulse oximeters with a view to improving patient care.

\section{Acknowledgements}

The authors gratefully acknowledge the secretarial assistance of Mrs. Sarah Scholl in the preparation of this manuscript. The authors also acknowledge the statistical expertise of Dr. Ian Shrier of the Centre for Clinical Epidemiology and Community Studies, Lady Davis Institute for Medical Research, SMBD-Jewish General Hospital, in the analysis of the sound pressure levels.

\section{References}

1 Schulte GT, Block FE Jr. Can people hear the pitch change on a variable-pitch pulse oximeter? J Clin Monit 1992; 8: 198-200.

2 Craven RM, McIndoe AK. Continuous auditory monitoring - how much information do we register? $\mathrm{Br} \mathrm{J}$ Anaesth 1999; 83: 747-9.

3 Santamore DC, Cleaver TG. The sounds of saturation. J Clin Monit 2004; 18: 89-92.

4 Shapiro RA, Berland T. Noise in the operating room. N Engl J Med 1972; 287: 1236-8. 
$5 \mathrm{Kam}$ PC, Kam AC, Thompson JF. Noise pollution in the anaesthetic and intensive care environment. Anaesthesia 1994; 49: 982-6.

6 Murthy VS, Malhotra SK, Bala I, Raghunathan M. Detrimental effects of noise on anaesthetists. Can J Anaesth 1995; 42: 608-11.

7 Hodge B, Thompson JF. Noise pollution in the operating theatre. Lancet 1990; 335: 891-4.

8 Wallace MS, Ashman MN, Matjasko MJ. Hearing acuity of anesthesiologists and alarm detection. Anesthesiology 1994; 81: 13-28.

9 Stevens JC. Acoustics. In: Kerr AG (Ed.). ScottBrown's Otolaryngology. $6^{\text {th }}$ ed. Oxford, UK:

Butterworth-Heinemann; 1997: 1-34.

10 “Sound." Encyclopaedia Britannica. 2003.

Encyclopaedia Britannica Online. Available from URL: http://www.search.eb.com/eb/article?eu=1 17555 (accessed 11 June, 2003), 2003.

11 Berger $K W$. Some factors in the recognition of timbre. J Acoust Soc Am 1966; 36: 1888-91.

12 Everest FA. Master Handbook of Acoustics, $4^{\text {th }}$ ed. New York, McGraw Hill Co; 2001.

13 Moore BC. An Introduction to the Psychology of Hearing, $5^{\text {th }}$ ed. San Diego: Academic Press; 2003. 